\title{
Estrategias para Compensar no Linealidades Generadas por Válvulas en Lazos de Control de Flujo
}

Rafael E. Vásquez y Fabio Castrillón

Universidad Pontificia Bolivariana, Escuela de Ingenierías, Grupo de Automática y Diseño A+D, Circular primera \# 70-01 bloque 11, A.A. 56006, Medellín-Colombia

(e-mail: rafavasquez@upb.edu.co; fcastrillon@upb.edu.co).

\begin{abstract}
Resumen
En este trabajo se compararon diferentes estrategias para compensar no linealidades generadas por válvulas en lazos de control de flujo. Se realizó la identificación de un proceso de flujo de líquido incom-presible mediante una prueba en planta y se desarrolló un sistema de control por computador. En este sistema fueron implementados un controlador lineal PI y cuatro diferentes estrategias de compensación, usando LabVIEW ${ }^{\circledR}$. La comparación fue realizada usando como índices de desempeño la integral del valor absoluto del error, el tiempo de estabilización y el sobrepaso. Las estrategias usadas permitieron mejorar el desempeño del controlador lineal en la zona de saturación del actuador. En conclusión, se puede considerar el uso de estos algoritmos para reemplazar elementos como controladores con auto-sintonía o posicionadores no lineales que elevan los costos de inversión y mantenimiento de la planta.
\end{abstract}

Palabras clave: válvulas de control, control no-lineal-control lineal, control de flujo, control computarizado

\section{Strategies to Compensate Non-Linearities Generated by Valves in Flow Control Loops}

\begin{abstract}
In this work, different strategies to compensate nonlinearities generated by valves in flow control loops were compared. The identification for an incompressible flow process was made through a plant test and a computer control system was developed. In this system a PI linear controller and four different compen-sation strategies were implemented using LabVIEW ${ }^{\mathrm{TM}}$. The comparison was made using the integral of the absolute value of the error, settling time and overshoot as performance indexes. The compared strategies allowed improving the performance of the linear controller in the actuator saturation zone. In conclusion, using these algorithms can be considered to replace elements as self-tuning controllers or nonlinear positioners that raise the inversion and maintenance costs of the plant.
\end{abstract}

Keywords: control valves, nonlinear control, linear control, flow control, computer controlled systems 


\section{INTRODUCCIÓN}

Los sistemas automáticos de control que se encuentran comúnmente en la industria de procesos químicos, cuentan con elementos lógicos de control diseñados para operar en sistemas lineales. Un alto porcentaje de la industria de procesos usa controladores tipo PID, dentro de los lazos de control, para mantener las variables de proceso en un valor de operación o punto de control, (Aström y Hägglund, 2001; Chen y Seborg, 2002).

Es deseable la presencia de una respuesta lineal en el proceso, ya que el desempeño de un controlador clásico por retroalimentación será similar para todo el rango de operación. La mayoría de los procesos cuentan con sistemas no lineales, como el caso de un proceso de control de flujo con una válvula que tiene una característica instalada no lineal (Ruel, 2000; Wilton, 2000), siendo necesario, realizar algunos tipos de compensación (Hägglund, 2002).

El Grupo de Investigación en Automática y Diseño $A+D$ de la Universidad Pontificia Bolivariana UPB - Medellín, ha implementado un proceso industrial a escala de laboratorio que sirve de plataforma para estudio y desarrollo de sistemas de control no lineal.

La planta caso de estudio es la red de fluidos del laboratorio de Ingeniería Mecánica de la UPB, instrumentada para la realización de mediciones de flujo volumétrico y presión en tuberías y adaptada para labores de control por Vásquez (2004). El elemento final de control del sistema es una válvula de tipo apertura rápida, responsable de la no linealidad del proceso. Se ha observado que un controlador convencional para el flujo volumétrico no presenta un buen desempeño en la zona de saturación del actuador, debido a la baja ganancia del proceso.

Una solución a este problema consiste en cambiar la característica no lineal de la válvula, lo cual puede lograrse por diferentes métodos, dado que existen posibilidades diferentes al uso de posicionadores no lineales. En su lugar pueden ser empleados bloques de cómputo o caracterizadores entre el controlador y la válvula, que desarrollen la función o característica requerida. Además pueden ser aplicadas ciertas estrategias sencillas del control no lineal.
Las cuatro estrategias que se comparan entre sí, permiten compensar la no linealidad generada por la válvula y son implementadas como bloques de cómputo dentro del sistema de control. Se usan como índices de desempeño: la integral del valor absoluto del error (IAE), tiempo de estabilización (Ts) y el sobrepaso (Mp).

\section{DESCRIPCIÓN Y MODELACIÓN DEL PROCESO DE FLUJO}

En la Fig. 1 se puede observar un diagrama de instrumentación y tubería que representa el proceso de flujo (para simplificación no se muestra la alimentación de los instrumentos).

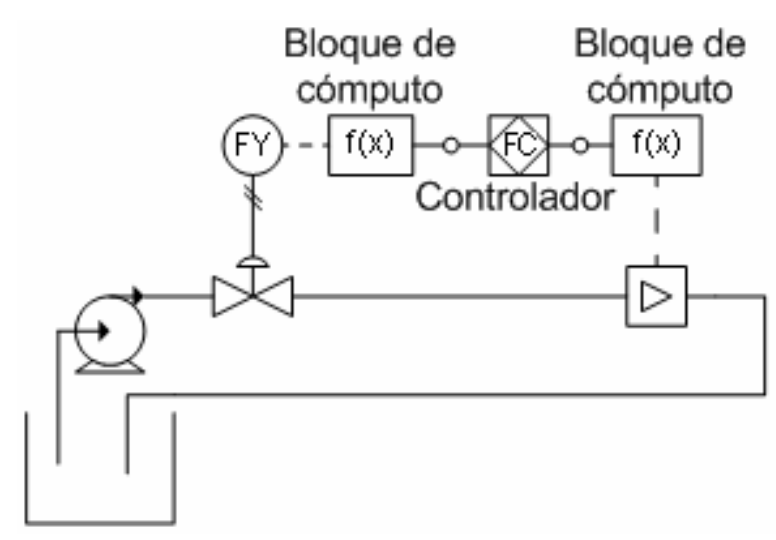

Fig. 1: Diagrama de instrumentación y tubería.

\section{Modelación del sistema}

Para determinar la linealidad o no linealidad estática de la planta, basta obtener la curva de estado estacionario del sistema. Se hacen cambios, de pequeña magnitud, en la salida del controlador, esperando a que la variable controlada (flujo volumétrico) se estabilice. Esto se repite hasta barrer todo el rango.

En la Fig. 2 se muestra la curva de estado estacionario obtenida a partir de cambios realizados de forma ascendente en la salida del controlador. La no linealidad obedece a que la característica inherente de la válvula es del tipo apertura rápida, lo que genera una característica instalada del mismo tipo pero más pronunciada.

Apertura rápida no indica que la válvula se abra en un periodo de tiempo corto, sino que para el primer $50 \%$ del rango de la señal de control la válvula se abre casi por completo y para la segunda mitad, la variación de apertura (y, por ende, de caudal) es ínfima. 


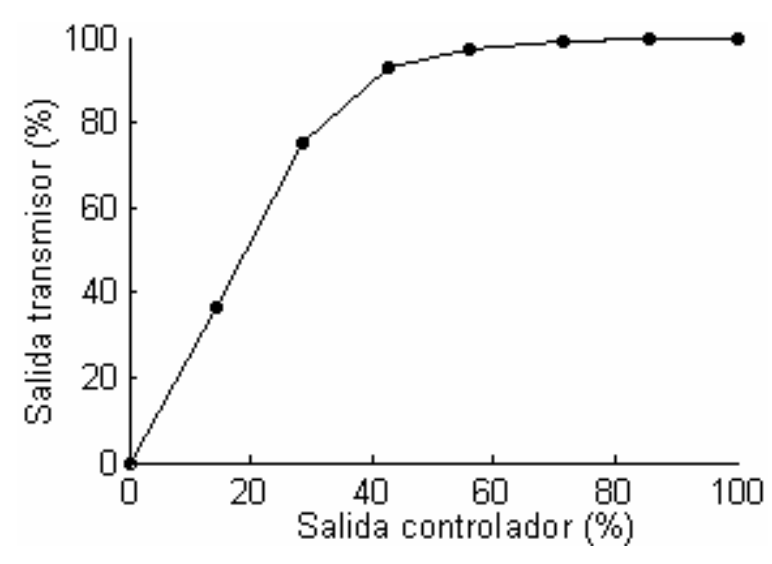

Fig. 2: Curva de estado estacionario.

Lo anterior implica una ganancia que varía considerablemente, siendo muy grande al inicio del rango y casi cero al final. Sin embargo, hay un rango estrecho de caudales, alrededor de 50 gpmUS, donde la ganancia varía muy poco, por lo cual se escoge como punto de operación para fines de diseño de un controlador lineal.

La ganancia de la planta se calcula promediando los valores de pendiente discreta entre tres puntos consecutivos alrededor del valor seleccionado como el punto de operación, $\mathrm{K}=17.835 \mathrm{gpmUS} / \mathrm{mA}$.

Para la caracterización dinámica de la planta, se realizó un escalón ascendente en la salida del controlador en lazo abierto alrededor del punto de operación elegido, Fig. 3.

En la prueba se observó que el sistema corresponde a un orden superior más tiempo muerto, pero que puede aproximarse por medios grafo-analíticos a un modelo primer orden más tiempo muerto. Este modelo abarca la dinámica del conversor digital análogo, del convertidor de corriente a presión, de la válvula, el retardo de transporte del proceso, la dinámica del sensor y del conversor análogo digital.

De la Fig. 3 se obtiene el modelo de primer orden más tiempo muerto (POMTM), con el método de los dos puntos, (Smith y Corripio, 1997).

$\mathrm{t}_{1}=\mathrm{t}_{\mathrm{o}}+\tau$

$\mathrm{t}_{2}=\mathrm{t}_{\mathrm{o}}+\tau / 3$
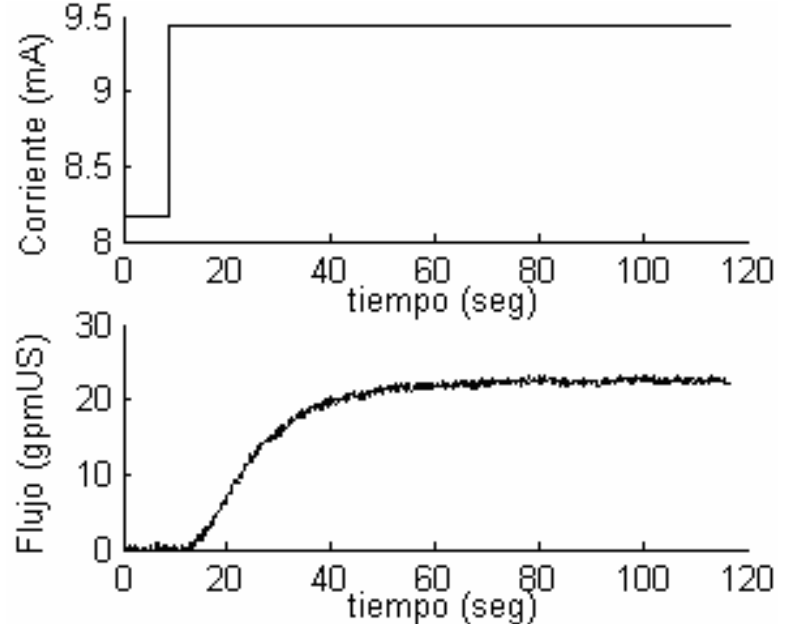

Fig. 3: Prueba dinámica en lazo abierto.

Los instantes de tiempo $t_{1}$ y $_{2}$ se miden a partir del momento en que se realizó el escalón y corresponden a los valores de $28.3 \%$ y $63.2 \%$ del cambio total en la salida. Los resultados para la gráfica ascendente son: $t_{0}=6.88 \mathrm{seg}$, $\tau=11.65$ seg.

Por tanto, el modelo de la planta alrededor del punto de operación en el dominio de Laplace es:

$G p(S)=\frac{17.835}{11.65 \cdot S+1} e^{-6.88 \cdot S}$

El diagrama de bloques se simplifica como se muestra en la Fig. 4.

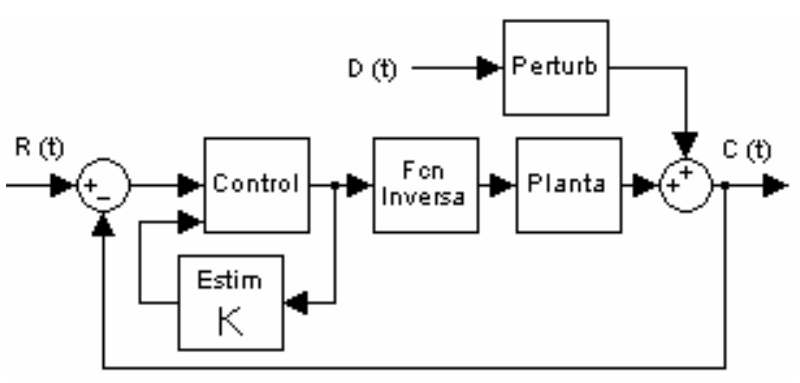

Fig. 4: Diagrama de bloques simplificado.

\section{ESTRATEGIAS DE CONTROL}

Síntesis del controlador

Los procesos de control de flujo en redes de tubería responden rápidamente. La dinámica presente en el proceso se debe principalmente a elementos tales como la válvula de control y el transmisor de flujo. Para este tipo de sistemas, según la práctica del control de procesos químicos, se recomienda un controlador PI, (Seborg, et al, 2003). 
La parte integral se hace necesaria para eliminar el error de estado estacionario, mientras que la parte derivativa se omite debido a que el flujo que circula por el interior de una tubería industrial, es generalmente turbulento y desordenado, lo cual genera una señal retroalimentada fluctuante, aún en condiciones de flujo estable. Como es bien sabido, la acción derivativa del controlador tiende a amplificar las fluctuaciones rápidas y el ruido de la señal retroalimentada, comprometiendo la estabilidad de la respuesta en lazo cerrado, lo que podría demostrase con un análisis de respuesta en frecuencia (Smith y Corripio, 1997).

$$
m(t)=\bar{m}+k_{p} \cdot e(t)+\int k_{i} \cdot e(t) \cdot d t
$$

Es de anotar que en (4) la constante integral se deja al interior del operador, con el fin de suavizar los cambios de la acción integral cuando se tiene un valor de $\mathrm{K}_{\mathrm{i}}$ variable, caso del controlador con ganancias programadas (Khalil, 2001). Esta es una buena práctica en el momento de la implementación ya que el algoritmo de control tiene cierta acción de filtrado que le permite mejorar su desempeño ante una variable con cambios aleatorios y rápidos (flujo de líquido). La ecuación en diferencias que permite digitalizar controlador está dada por:

$m(k)=\bar{m}+K_{p} \cdot e(k)+\sum_{j=1}^{k} T \cdot K_{i}(j) \cdot e(j)$

Para la sintonía del controlador se usó el método de síntesis directa utilizando los lineamientos de Seborg, et al. (2003), el cual se desarrolla para operación servo, teniendo en cuenta que la mayoría de los lazos de control de flujo trabajan como esclavos dentro de sistemas en cascada (Shinskey, 2000).

$$
\begin{aligned}
& \mathrm{K}_{\mathrm{p}}=\frac{\tau}{\mathrm{K}\left(\mathrm{t}_{\mathrm{o}}+\tau_{\mathrm{c}}\right)} \\
& \mathrm{K}_{\mathrm{i}}=\frac{1}{\mathrm{~K}\left(\mathrm{t}_{\mathrm{o}}+\tau_{\mathrm{c}}\right)}
\end{aligned}
$$

Donde $\tau_{c}$ : es la constante de tiempo para el sistema automático de control en operación servo.
El valor de $\tau_{\mathrm{C}}$ fue establecido de acuerdo al siguiente lineamiento (Seborg, 2003): $t_{0}<\tau_{C}<\tau$. Los valores obtenidos fueron: $\mathrm{Kp}=0.14 \mathrm{~mA} / \mathrm{mA}$ y Ki=0.012 rep/seg

\section{Función Inversa}

El método de la función inversa consiste en utilizar un caracterizador de funciones, implementado en un bloque de cómputo que se inserta a la salida del controlador, Fig. 4, con el fin de simular la función inversa de la planta.

Se pretende linealizar una válvula de apertura rápida y el caracterizador debe tomar una forma opuesta a la característica de la válvula, Fig. 5. Debido a la zona de saturación que presenta la válvula, su función inversa presenta una zona de alta ganancia para valores altos de salida del transmisor. Se pueden vislumbrar problemas en la implementación para la variable controlada del proceso, la cual es ruidosa por naturaleza y presenta cambios súbitos cada vez más pronunciados a medida que aumenta el flujo.

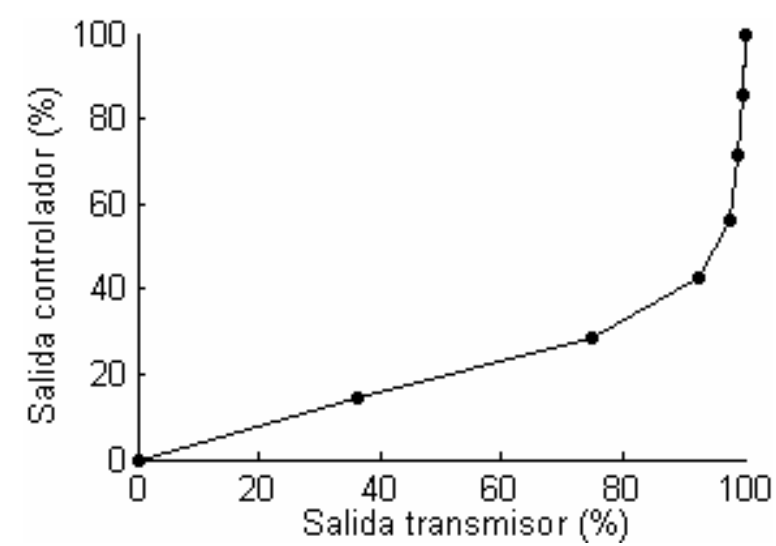

Fig. 5: Función inversa del proceso.

La implementación se hace en LabVIEW ${ }^{\circledR}$ mediante un bloque de cómputo que interpola racionalmente en tiempo real, durante cada periodo de muestreo, y modifica la salida del controlador mediante la curva de la función inversa para linealizar el proceso.

\section{Caracterizador Universal}

El método de caracterizador universal ajusta una función hiperbólica a la función inversa de la válvula de control, para este caso:

$f(m)=\frac{m}{L+(1-L) \cdot m}$ 
Donde $m$ es la salida del controlador y $L$ es un parámetro ajustable que determina la linealidad de la función. Es decir, si $L$ es igual a uno, quiere decir que la función es lineal, mientras que los valores de $L$ que son mayores o menores que uno le dan la forma a la curva en direcciones opuestas.

El parámetro L que utiliza esta función se ajustó por medio de regresión no lineal utilizando mínimos cuadrados. En la ecuación (8), $m$ es la salida del controlador y $f(m)$ la señal que llega a la válvula de control. Un ajuste inicial realizado por mínimos cuadrados arrojó un valor para $L=22$, pero el sistema se indetermina con valores de la salida del controlador muy cercanos de $1(m=1.04)$ que son factibles debidos al ruido. Además, este valor genera una pendiente alta en la zona de saturación de la válvula, por lo tanto se usó un valor más conservador que no garantiza el ajuste preciso, pero que se torna menos agresivo en las regiones de alto flujo. Se tomó un valor de $\mathrm{L}=10$ y se evaluó la función para el rango de salida del transmisor en fracción, con lo cual se implementó un bloque de cómputo en LabVIEW ${ }^{\circledR}$ similar al bloque generado para la función inversa.

\section{Controlador PI no lineal}

Una posible alternativa para compensar la presencia de no linealidades en el lazo es modificar el PI convencional, de forma tal que sea capaz de encontrar la manera de salir lo más rápido posible de la zona no lineal, en la cual se encuentra el sistema siempre que se aleja del punto de operación. Para tal efecto puede hacerse una modificación a la estructura convencional del PI, para que la ganancia sea proporcional al error (PI de error cuadrático).

Los autores realizaron una modificación al algoritmo normalmente propuesto en la literatura, (Shinskey 1994; Smith y Corripio, 1997; Corripio, 2001).

$m(t)=\bar{m}+\frac{|c(t)|}{100}\left(K_{p} \cdot e(t)+\int k_{i} \cdot e(t) \cdot d t\right)$

En este caso la sensibilidad del controlador a los errores no depende directamente del error, sino del valor absoluto de la variable controlada. A medida que aumenta el flujo, disminuye la ganancia del proceso, y se nece- sita una acción más agresiva del controlador, lo cual se logra cuando su salida es proporcional al valor de la variable controlada con un valor alto.

Para valores pequeños de $|c(t)|$, la ganancia del proceso es alta y por ende se necesita menos acción de control para eliminar el error. Con la propuesta se evita además el rebote proporcional al realizar cambios ascendentes del punto de control. La implementación se hizo en LabVIEW®.

\section{Controlador PI con ganancias programadas}

El método de ganancias programadas se caracteriza por modificar los parámetros proporcional e integral, de acuerdo a los valores que tome la ganancia del proceso en cada punto de operación (Khalil, 2001; Wang y Crusca, 2002). Lo anterior se logró ligando los valores de la ganancia del proceso, Fig. 6, con la señal de salida del controlador, de tal manera que para cada valor se determina la ganancia del pro-ceso y se actualizan los parámetros del con-trolador, (6) y (7), durante cada instante de muestreo.

Para el diseño del algoritmo se siguieron los lineamientos de Khalil (2001) y Stephanopoulos (1984). La implementación se hizo mediante un bloque de cómputo en LabVIEW ${ }^{\circledR}$.

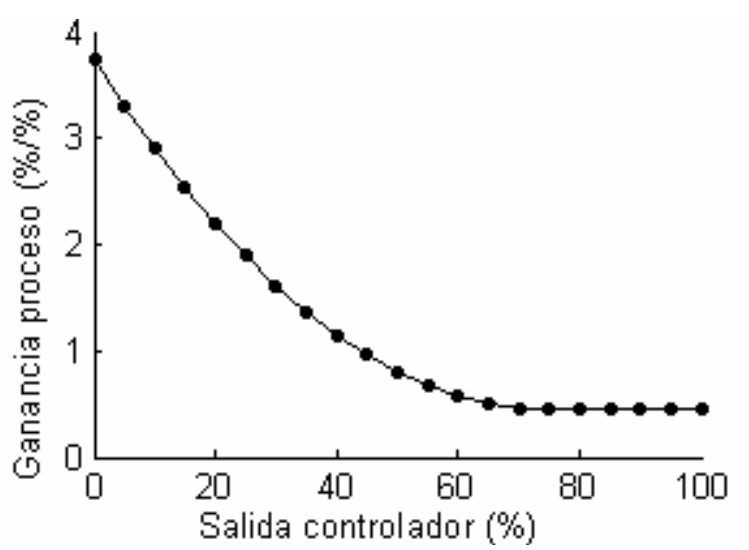

Fig. 6: Ganancia del proceso v/s salida del controlador

\section{RESULTADOS}

Para la evaluación de las diferentes estrategias de compensación, se hicieron dos pruebas tipo escalón en la referencia para evaluar la operación servo del sistema de control. 
Prueba del tipo escalón en la referencia, valor inicial de operación 50 gpmUS, valor final de operación 65 gpmUS. De la Fig. 7 a la Fig. 11 se presentan la salida del controlador y la variable controlada en función del tiempo.

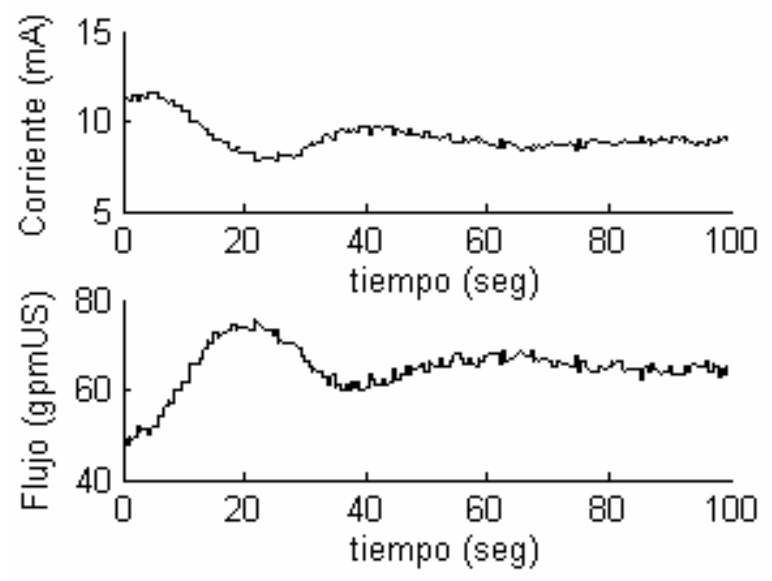

Fig. 7: Controlador PI Lineal. $\mathrm{Kp}=0.14 \mathrm{~mA} / \mathrm{mA}$ $\mathrm{Ki}=0.012 \mathrm{rep} / \mathrm{seg}$.

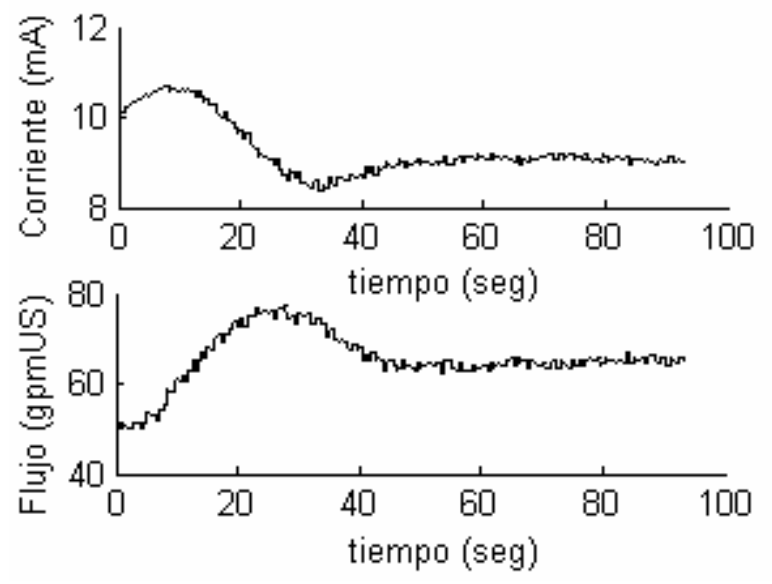

Fig. 8: Controlador $\mathrm{PI}$ No lineal. $\mathrm{Kp}=0.14$ $\mathrm{mA} / \mathrm{mA} \mathrm{Ki}=0.012 \mathrm{rep} / \mathrm{seg}$.

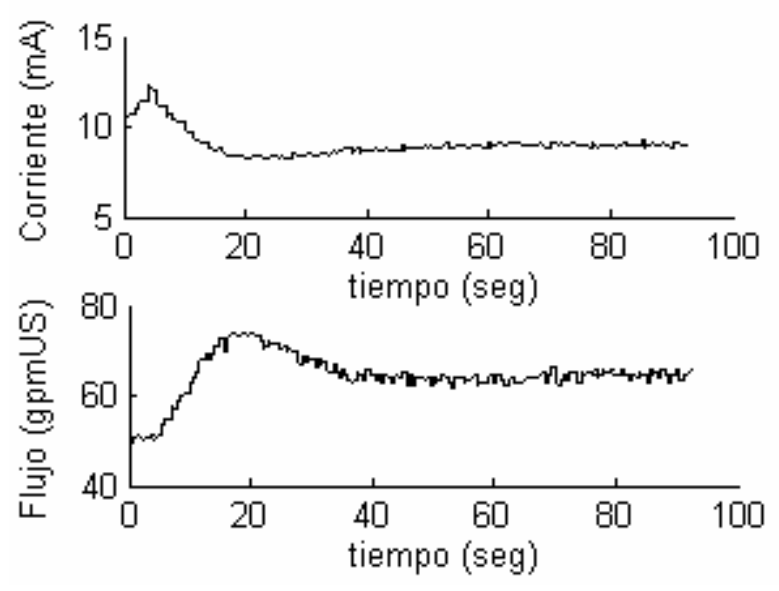

Fig. 9: Bloque de cómputo con la función inversa. $\mathrm{Kp}=0.14 \mathrm{~mA} / \mathrm{mA} \mathrm{Ki=0.012} \mathrm{rep/seg.}$

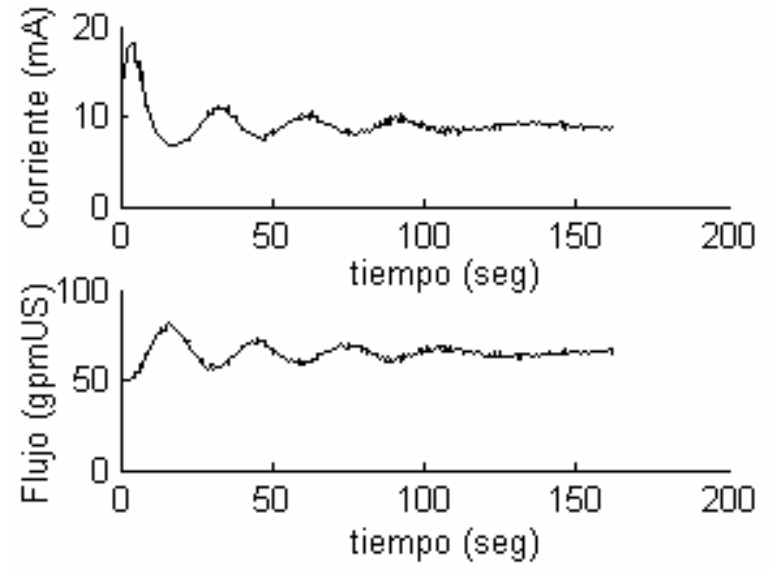

Fig. 10: Bloque de cómputo con caracterizador universal. $\mathrm{Kp}=0.14 \mathrm{~mA} / \mathrm{mA} \mathrm{Ki}=0.012$ rep/seg.

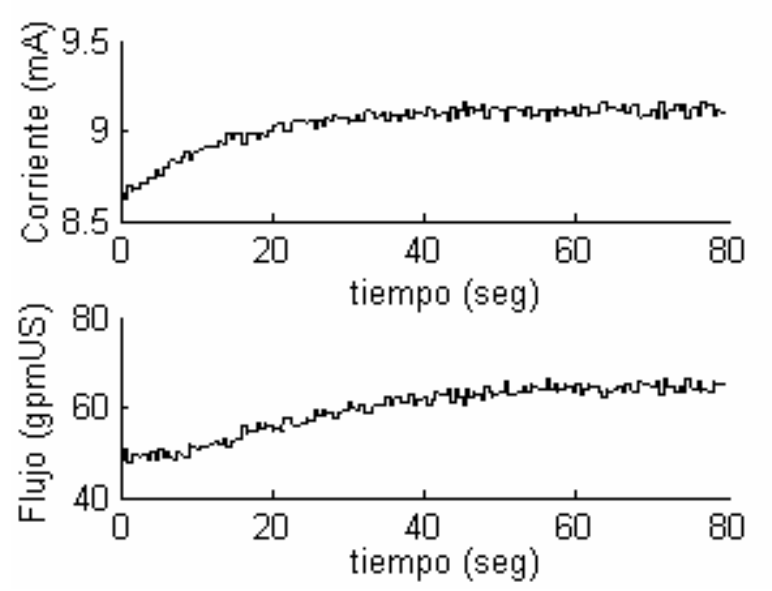

Fig. 11: Controlador PI con Ganancias Programadas.

La Tabla 1 muestra los índices de desempeño obtenidos con cada una de las estrategias para la prueba del tipo escalón en la referencia, valor inicial de operación 50 gpmUS, valor final de operación 65 gpmUS.

Tabla 1. Comparación de las diferentes estrategias de control para la operación Servo entre 50 y 65 gpmUS.

\begin{tabular}{|l|l|l|l|}
\hline & $\begin{array}{l}\text { IAE } \\
\left(\mathrm{gpm}^{\star} \mathrm{seg}\right)\end{array}$ & $\begin{array}{l}\mathrm{Mp} \\
(\%)\end{array}$ & $\begin{array}{l}\text { Ts } \\
(\mathrm{seg})\end{array}$ \\
\hline PI Lineal & 357.33 & 70.11 & 114 \\
\hline PI No lineal & 365.13 & 83.2 & 87 \\
\hline Función Inversa & 283.24 & 61.05 & 82 \\
\hline $\begin{array}{l}\text { Caracterizador } \\
\text { Universal }\end{array}$ & 578.75 & 111.69 & 156 \\
\hline $\begin{array}{l}\text { Ganancias } \\
\text { programadas }\end{array}$ & 421.49 & 4 & 65.5 \\
\hline
\end{tabular}

Información Tecnológica - Vol. 18 No 1 - 2007 
Prueba del tipo escalón en la referencia, valor inicial de operación 80 gpmUS, valor final de operación 50 gpmUS. De la Fig. 12 a la Fig. 16 se presentan la salida del controlador y la variable controlada en función del tiempo.

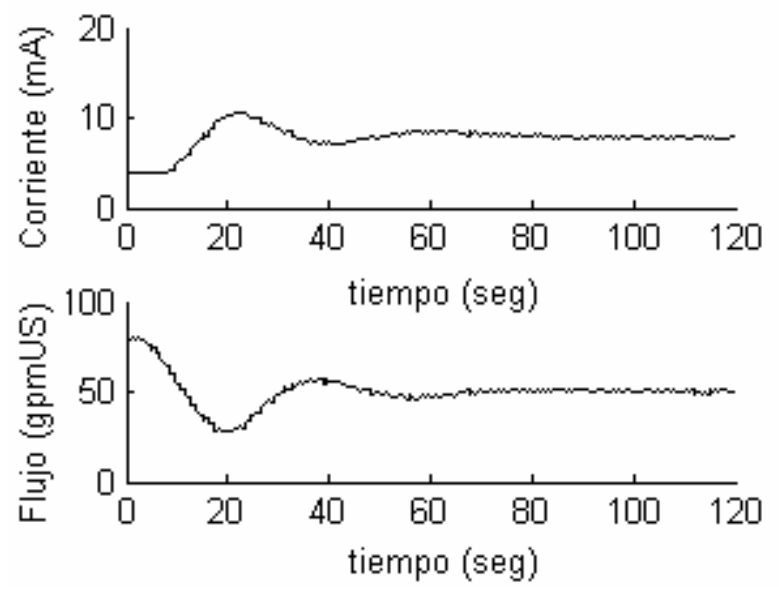

Fig. 12: Controlador PI Lineal. $\mathrm{Kp}=0.14$ $\mathrm{mA} / \mathrm{mA} \mathrm{Ki}=0.012 \mathrm{rep} / \mathrm{seg}$.

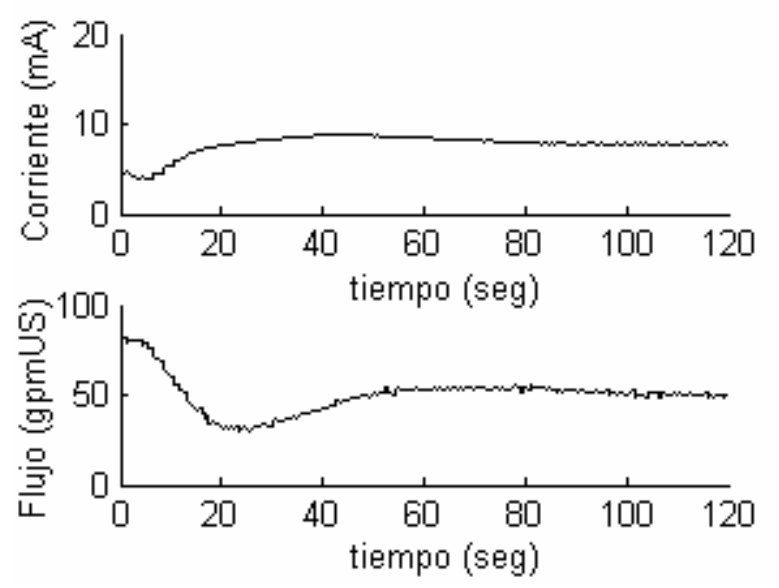

Fig. 13: Controlador $\mathrm{PI}$ No lineal $\mathrm{Kp}=0.14$ $\mathrm{mA} / \mathrm{mA} \mathrm{Ki}=0.012 \mathrm{rep} / \mathrm{seg}$.

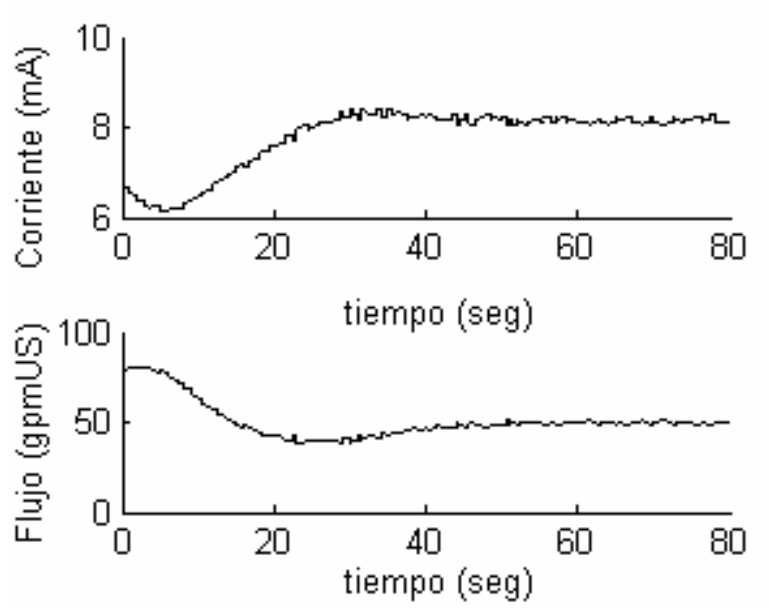

Fig. 14: Bloque de cómputo con la función inversa. $\mathrm{Kp}=0.14 \mathrm{~mA} / \mathrm{mA} \mathrm{Ki=0.012} \mathrm{rep/seg.}$

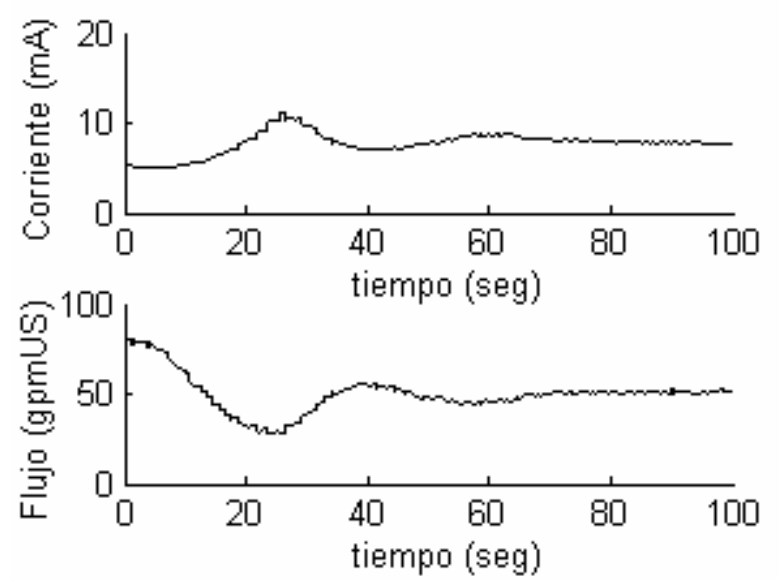

Fig. 15: Bloque de cómputo con caracterizador universal. $\mathrm{Kp}=0.14 \mathrm{~mA} / \mathrm{mA} \mathrm{Ki}=0.012$ rep/seg.

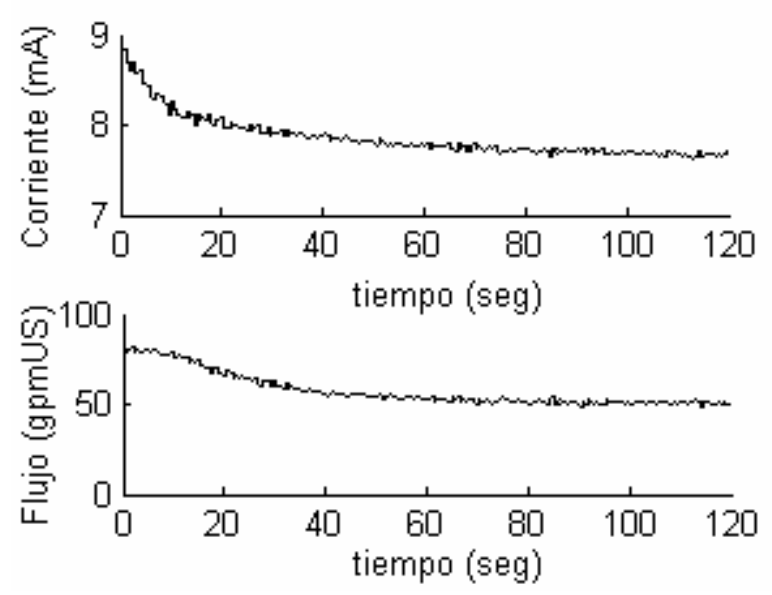

Fig. 16: Controlador PI con Ganancias Programadas.

La Tabla 2 muestra los índices de desempeño obtenidos con cada una de las estrategias para la prueba del tipo escalón en la referencia, valor inicial de operación 80 gpmUS, valor final de operación 50 gpmUS.

Tabla 2. Comparación de las diferentes estrategias de control para la operación Servo entre 80 y 50 gpmUS.

\begin{tabular}{|l|l|l|l|}
\hline & $\begin{array}{l}\text { IAE } \\
(\mathrm{gpm} * s e g)\end{array}$ & $\begin{array}{l}\mathrm{Mp} \\
(\%)\end{array}$ & $\begin{array}{l}\text { Ts } \\
(\mathrm{seg})\end{array}$ \\
\hline PI Lineal & 637.84 & 73.75 & 124 \\
\hline PI No lineal & 811.2 & 66.87 & 117.5 \\
\hline Función Inversa & 499.9 & 37.95 & 63 \\
\hline $\begin{array}{l}\text { Caracterizador } \\
\text { Universal }\end{array}$ & 647.97 & 71.61 & 82.5 \\
\hline $\begin{array}{l}\text { Ganancias } \\
\text { programadas }\end{array}$ & 897.27 & 2.45 & 100.5 \\
\hline
\end{tabular}




\section{CONCLUSIONES}

El controlador PI lineal arroja, en términos generales, valores bajos de IAE, lo cual muestra su agresividad frente a los errores, pero el sobrepaso y el tiempo de estabilización son medios y elevados frente a cambios en el punto de control. Su salida es bastante ruidosa lo que compromete la vida útil del actuador.

La Función Inversa arroja los menores valores de IAE en operación servo y reguladora, excepto para valores de la variable controlada contenidos dentro de la zona de saturación de la válvula de control. En dicha zona la función inversa presenta un comportamiento asintótico. Lo anterior produce cambios bruscos en la salida, que generan saturación del controlador.

El Caracterizador Universal tiene un rendimiento bajo, ya que proporciona respuestas demasiado oscilatorias con altos valores de sobrepaso. Para valores de la variable controlada contenidos dentro de la zona de saturación de la válvula de control, presenta una alta probabilidad de saturar la salida del controlador.

El controlador PI no lineal tiene rendimiento moderado, ya que muestra valores intermedios de los índices de comparación. Cabe resaltar su capacidad de filtrado y las suaves señales de corrección que entrega, beneficiando la vida útil de la válvula de control.

El controlador PI con ganancias programadas presenta una respuesta que es independiente del tamaño del cambio realizado en el punto de control, lo cual garantiza repetibilidad en el comportamiento del sistema. Proporciona los menores valores de tiempo de estabilización y sobrepaso, frente a cambios en el punto de control. La señal de corrección que entrega siempre es suave. Presenta cierto carácter predictivo pues estabiliza la señal de salida del controlador antes de que la variable esté posicionada en el punto de control.

Los resultados obtenidos, muestran que se pueden usar estrategias de compensación para las no linealidades en lazos de control de flujo, en lugar de usar controladores con autosintonía o posicionadores no lineales que elevan los costos de inversión y mantenimiento de la planta.

\section{REFERENCIAS}

Aström, K. y T. Hägglund, Future of PID Control, Control Engineering Practice, 9(11), 1163-1175 (2001).

Chen, D. y D. Seborg, PI/PID Controller design based on direct synthesis and disturbance rejection, Ind. Eng. Chem. Res., 41(19), 4807-4822 (2002).

Corripio A., Tuning of industrial control systems, $2^{a}$ edición, ISA, NC, USA (2001).

Hägglund, T., A friction compensator for pneumatic control valves, Journal of Process Control, (12), 897-904 (2002).

Khalil, H., Nonlinear Systems, $3^{\mathrm{a}}$ edición, Prentice Hall, Nueva York, USA (2001).

Ruel, M., How valve performance affects the control loop, Chem. Eng. 107(10), (2000).

Seborg, D., T. Edgar. y D. Mellichamp, Process dynamics and control, $2^{\mathrm{a}}$ edición., John Wiley \& Sons, New York, USA (2003).

Shinskey, F., Feedback Controllers for the Process Industries, McGraw Hill, Singapur (1994).

Shinskey, F., Optimización de la regulación de la carga, Ingeniería Química, (366), (2000).

Smith, C. y A. Corripio, Principles and practice of automatic process control, $2^{\mathrm{a}}$ edición., John Wiley \& Sons, New York, USA (1997).

Stephanopoulos, G., Chemical process control, Prentice Hall, New Jersey, USA (1984).

Vásquez, J. y R. Vásquez, Implementación de un control digital en un PC para un proceso de flujo, Memorias del VI Congreso Asociación Colombiana de Automática, Ibagué, Colombia (2004).

Wang, M. y F. Crusca, Design and implementation of a gain scheduling controller for a level control system, ISA transactions, 41(3), (2002).

Wilton, S., Control valves and process variability, ISA transactions, 39(2), 265-271 (2000).

Información Tecnológica - Vol. 18 No 1 - 2007 